\title{
BMJ Open Geographical variations and factors associated with recent HIV testing prevalence in Ghana: spatial mapping and complex survey analyses of the 2014 demographic and health surveys
}

\author{
Jerry John Nutor (D) , ${ }^{1}$ Henry Ofori Duah (D) , ${ }^{2}$ Precious Adade Duodu, ${ }^{3}$ \\ Pascal Agbadi (D) , ${ }^{4}$ Robert Kaba Alhassan, ${ }^{5}$ Ernest Darkwah ${ }^{6}$
}

To cite: Nutor JJ, Duah HO, Duodu PA, et al. Geographical variations and factors associated with recent HIV testing prevalence in Ghana: spatial mapping and complex survey analyses of the 2014 demographic and health surveys. BMJ Open 2021;11:e045458. doi:10.1136/ bmjopen-2020-045458

- Prepublication history and additional supplemental material for this paper are available online. To view these files, please visit the journal online (http://dx.doi.org/10.1136/ bmjopen-2020-045458).

Received 04 0ctober 2020 Accepted 22 June 2021

Check for updates

(C) Author(s) (or their employer(s)) 2021. Re-use permitted under CC BY-NC. No commercial re-use. See rights and permissions. Published by BMJ.

For numbered affiliations see end of article.

Correspondence to Dr Jerry John Nutor; Jerry.Nutor@ucsf.edu

\section{ABSTRACT}

Objective To examine the factors associated with recent HIV testing and to develop an HIV testing prevalence surface map using spatial interpolation techniques to identify geographical areas with low and high HIV testing rates in Ghana.

Design Secondary analysis of Demographic and Health Survey.

Setting Rural and urban Ghana

Participants The study sample comprised 9380 women and 3854 men of 15-49 years.

Results We found that $13 \%$ of women and $6 \%$ of men of Ghana had tested for HIV in the past 12 months. For women, being within the age groups of 15-39 years, being currently married, attainment of post-secondary education, having only one sexual partner and dwelling in certain regions with reference to greater Accra (Volta, Eastern, Upper West and Upper East) were associated with a higher likelihood of HIV testing. For men, being older than 19 years, attainment of post-secondary education and dwelling in the Upper East region with reference to the greater Accra region were significantly associated with a higher likelihood of HIV testing. The surface map further revealed intra-regional level differences in HIV testing estimates.

Conclusion Given the results, HIV testing must be expanded with equitable testing resource allocation that target areas within the regions in Ghana with low HIV testing prevalence. Men should be encouraged to be tested for HIV.

\section{INTRODUCTION}

Despite the progress made in advancing knowledge and antiretroviral treatment, the HIV continues to cause a high number of deaths and morbidity globally. ${ }^{1}$ Globally, about 37.9 million people were living with HIV in 2018. ${ }^{1}$ Sub-Saharan Africa (SSA) alone accounts for over $70 \%$ of people living with HIV, although it is home to only about $12 \%$ of the global population. ${ }^{3}$ Testing programmes may help to estimate the prevalence and
Strengths and limitations of this study

- This study used a large, nationally representative survey data set that employed a robust methodology for analysis.

- A large number of people in sub-Sharan Africa are unaware of their HIV status, thereby missing the opportunity for care and treatment.

- There are limited studies on spatial analysis to reveal intra-regional level differences in HIV testing in Ghana.

- The cross-sectional design of this study precluded any causal inference.

HIV testing must be expanded with equitable testing resource allocation that target areas within the regions in Ghana with low HIV testing.

predictors of the disease towards developing context-specific policy actions to combat the disease. However, a large number of people in SSA are unaware of their HIV status, thereby missing the opportunity for care and treatment. ${ }^{4}$

Knowing HIV status is the first and critical step towards eradicating AIDS. ${ }^{5}$ Therefore, the Joint United Nations Programme on HIV/AIDS (UNAIDS) set the '90-90-90 target' to be achieved by the end of $2020{ }^{6}$ The target has called for $90 \%$ of people living with HIV are to be aware of their HIV status, $90 \%$ of those diagnosed with HIV to have access to antiretroviral therapy (ART) and $90 \%$ of those receiving ART to achieve suppression of the viral loads. ${ }^{6}$ Sadly, the well-established HIV prevention tools are few amidst the mitigating factors affecting the desire for and uptake of HIV testing such as stigma, and therefore early diagnosis and early ART needs to be prioritised. ${ }^{7-9}$ The limitation of the HIV testing-care-treatment 
continuum may adversely affect the achievement of the global '90-90-90' target.

In Ghana, HIV is epidemiologically described as mature, mixed and generalised..$^{10}$ Recent studies report HIV prevalence of about $1.6 \%$ among the general population. ${ }^{112}$ The epidemic is largely driven by heterosexual contact and mother-to-child transmission, ${ }^{13}$ and also varies among groups and geographical locations. ${ }^{11} 14$ There are regional and geographical variations, with the highest prevalence reported in Eastern, Western, Greater Accra ${ }^{11}$ and Volta region, ${ }^{15}$ and the lowest in the three northern regions. ${ }^{11}$ Nevertheless, there is limited literature data on intra-regional variations in HIV testing rate in Ghana. This knowledge is important in identifying low testing zones in high testing regions and high testing zones in low testing regions for targeted testing interventions. In Ghana, HIV testing is offered in hospitals and clinics with testing compulsory for women attending antenatal clinics. It is necessary for citizens to know their HIV status in order to receive treatment and help curb the incidence of new infections. Therefore, the current study aimed to examine factors associated with HIV testing and spatial interpolation of the prevalence of HIV testing in Ghana using the nationally representative Demographic and Health Survey data. Understanding the geographical distribution of HIV testing will help public health officials and policymakers equitably distribute resources to areas that are less likely to be tested and to help reduce the spread of the virus.

\section{METHODS}

\section{Patient and public involvement}

Our study analysed publicly available secondary data (Ghana Demographic and Health Survey (GDHS) 2014) from the demographic and health surveys database. Thus, patients and the public were not involved.

\section{Study design}

This paper employed an analysis of secondary data using the 2014 GDHS. ${ }^{16}$ As a cross-country survey, the GDHS is conducted to assess the general health of the population with a special focus on maternal and child health indicators as well as other themes of global health importance such as the prevalence of HIV prevalence, testing and treatment. The 2014 GDHS data collection was operationalised by the Ghana Statistical Service (GSS) and the Ghana Health Service with funding from the US Agency for International Development and other international donors. Technical support was also provided by the ICF international. The census frame used for the 2014 GDHS consisted of all enumeration areas demarcated during the 2010 Ghana's Population and Housing Census. The 2014 GDHS was undertaken from early September to mid-December 2014. The 2014 GDHS adopted a multistage sampling in enrolling households and individuals. The first stage involved the random selection of clusters (enumeration areas). The second stage involved using systematic sampling to select households to be interviewed from clusters that had already been selected during the first stage. Sampling was also stratified to account for rural and urban variations. In all, 216 and 211 clusters were selected from urban and rural areas, respectively, making a total of 427 clusters. On average, about 30 households were chosen from each selected cluster constituting a total of 12831 selected households in the 2014 GDHS. The probability of cluster selection was proportional to the cluster size and independent at each sampling stratum. The probability of cluster selection was proportional to the size of the cluster size and independent at each sampling stratum.

\section{Study setting}

The study setting is the republic of Ghana. Ghana is a lower-middle-income country in West Africa with a population of about 25 million people at the time of the 2014 GDHS. It shares boundaries Togo on the east, Burkina Faso on the north and northwest, and Côte d'Ivoire on the west. $^{16}$

\section{Measurements}

The outcome variable under investigation was HIV testing in the past 12 months. This was assessed for each adult respondent in the survey by asking how long ago they had tested for HIV. For our study, we recoded the variable and grouped the cases who tested for HIV in the past 12 months as ' 1 ' and all others as ' 0 '. The following sociodemographic and behavioural factors were included are sex, age, education level, marital status, religion, the total number of sexual partners in the past 12 months, history of sexually transmitted infections (STIs), household wealth index, place and region of residence. The household wealth index was already calculated and reported in the DHS data. It was estimated using household socioeconomic indicators such as the main roof and floor material of households, type of toilet facilities, source of drinking water, source of domestic cooking fuel, possession of television, radio, vehicle, motorcycles, agricultural land, farm animals among other movable and immovable assets. The GDHS employed factor analysis to allot weights to every household asset and a cumulative score was calculated from the allotted weights. Households were graded according to the aggregate scores. Aggregate wealth scores were classified using a percentage distribution and categorised into quintiles using discrete cut points. Poorest households were defined as wealth scores less than or equal to the 20th percentile; poorer households were those with scores greater than 20th percentile but less than or equal to 40th percentile; middle households were those with aggregate scores greater than the 40th percentile and less than or equal to the 60th percentile score; households with aggregate scores greater than the 60 th percentile but less than or equal to the 80 th percentile while households with aggregate scores greater than the 80th percentile were assigned as richest households. 


\section{Data collection, access, preparation and analysis}

Data collection was done by trained enumeration officials from GSS. As part of the data collection, respondents were asked if they had ever undergone testing for HIV. Data on other sociodemographic variables were collected as described above. The 2014 GDHS data used for analysis in this study is easily accessible at www.dhsprogram.com and can be freely downloaded after an online request by individuals. Data were downloaded from the DHS programme website after permission was obtained by the primary author, initially prepared in SPSS and analysed using Stata V.16. The women and men data were separately downloaded comprising 9396 and 4388 cases, respectively. For the women data set, 16 cases with incomplete data on HIV testing and the covariates were dropped. Five hundred and thirty-three (533) men of 50 years and above were excluded from the analyses for men using 'subpop' function associated with the 'svy' command to allow a balanced comparison with the women. One case (1) with missing information on the outcome variable in the men data sets was excluded. The final sample sizes used for the analyses were 9380 women and 3854 men of 15-49 years. The key variables were selected and included in the final analysis using univariate, bivariate and multivariable approaches. We stratified bivariate analysis by gender and assessed for the presence of interaction effect of gender on the association between each study covariate and HIV testing among Ghanaians. The presence of significant interaction was assessed with the adjusted Wald Test. Multivariate estimates of the factors associated with HIV testing was performed separately for men and women samples.

The 'gvselect' - Best subsets variable selection-in Stata was used to identify the best features to build models that explain the variability in HIV testing among Ghanaian men and women samples. As the name suggests, the 'gvselect' performs best subsets variable selection. ${ }^{17}$ The variable selection is based on 'the Furnival-Wilson leapsand-bounds algorithm' published in $1974,{ }^{18}$ which is 'applied using the log-likelihoods of candidate models, allowing variable selection to be performed on a wide family of normal and non-normal regression models'. ${ }^{17}$ This method is described in the works of Lawless and Singhal published in $1978 .{ }^{19}$ The log-likelihood, Akaike's information criterion (AIC), and the Bayesian information criterion are reported for the best regressions at each predictor quantity. ${ }^{17}$ Thus, the model with the lowest AIC value is preferred. These variables (with their labels) were included in the 'gvselect' equation: aaaV013 (age), aaV106 (Education), aaaV502 (marital status), Religyn (religion), SexPart (sex partners including partner in last 12 months), aaaV763A (history of STI), aaaV190 (household wealth index), aaaV025 (urban-rural residence), aaV024 (region of residence) (online supplemental files 1 and 2). Coefficient plots for both the women and men models were generated using the 'coefplot', which is used for plotting regression coefficients.
We accounted for sample weight in the univariate and bivariate analysis. In the multivariate analysis, we used a complex survey design in Stata to account for sampling design. This was achieved using the 'svyset' command to account for clusters or primary sampling units $(n=427)$, sample strata $(n=20)$ and sample weights. We used a generalised linear model with family set to 'Poisson' to report prevalence ratio (PR) estimates instead of using a standard logistic regression used to report the OR. We reported both crude and adjusted PRs (APRs).

\section{Spatial analysis}

We also performed spatial analysis to visualise HIV testing at the subregional level using clusters as the focus of the analysis. This was done using the prevR package in the $\mathrm{R}$ freeware for statistical analysis. ${ }^{20}$ This package was specifically developed to perform spatial estimation of regional trends of a prevalence using data from complex surveys involving two-staged sampling. ${ }^{20}$ With the aid of the functions available in the prevR package, we used the kernel estimator approach with adaptive bandwidths of an equal number of persons surveyed to produce a surface of HIV testing prevalence. ${ }^{20}$ The main surface is an estimated HIV testing surface with parameter ( $\mathrm{N}=233$ for women sample and $\mathrm{N}=201$ for men sample), a value that is chosen using the Noptim() function in the prevR package. ${ }^{20}$ The maps with the smoothing circle radius contours (in kilometres) have been added as supplementary figures (Women: online supplemental figure 1, Men: online supplemental figure 2). We used the 'foreign' package in $\mathrm{R}$ to read the data in R whiles using 'ggplot2' and 'maptools' packages to demonstrate the HIV testing prevalence map. Spatial analysis was done using R V.3.5.3. ${ }^{21}$

\section{Ethical considerations}

Enumerators obtained consent for enrolment from all respondents on behalf of GSS and the DHS programme. We did not obtain any further consents. The application of spatial maps raises concerns for potential identification of respondents in their households on maps, especially for a sensitive topic such as HIV testing in West Africa. However, this was addressed as the spatial data included only the Global Positioning System (GPS) coordinates of the centre points of the clusters instead of the actual location of individual households. Moreover, GPS coordinates of the centre points of the clusters were displaced at a random angle by up to $2 \mathrm{~km}$ and $5 \mathrm{~km}$ for urban and rural clusters, respectively. Additionally, GPS locations for about $1 \%$ of the rural clusters were displaced by $10 \mathrm{~km}$. This helps to ensure that the actual households will not be identifiable on maps, but the trade-off is that it makes the spatial analysis less accurate.

\section{RESULTS}

\section{Sample description}

Table 1 describes the women and men samples, highlighting the association between HIV testing in the past 
12 months and sociodemographic factors. In all, 15-49 years old women (9380 cases) and men (3854 cases) were included in the analysis. In terms of quinary age distribution, the women showed equal proportion for the first three quinary age groups (17\% each), with a gradual decline in the proportions with increasing age in the subsequent quinary age groups. Conversely, $22 \%$ of the men were aged $15-19$ years, $15 \%$ each for the $20-25$ years and 26-30 years groups and gradual decline in proportion with increasing age in the subsequent quinary age groups just like the pattern observed among the women. About $57 \%$ and $65 \%$ of women and men had attained secondary education, respectively. Approximately 33\% and $48 \%$ of the women and men had never been in a union at the time of the survey, respectively. Religious affiliation was mainly Pentecostal/Charismatic among the women $(41 \%)$ and men $(31 \%)$. Only $1 \%$ of the women had had more than one sexual partner within the last 12 months preceding the survey compared with $14 \%$ among their men counterparts. More women (54\%) than men $(53 \%)$ resided in urban areas.

About $13 \%$ of the women had screened and received results for HIV testing in the last 12 months preceding the survey compared with $6 \%$ in the men sample. For women, the following subgroups had a higher HIV screening rate compared with the national women average of 13\%: Those aged 25-29 years (19\%) and $30-34$ years $(19 \%)$, women currently married $(16 \%)$, those with previous STI (16\%), women with only one sexual partner (16\%), from richest households $(18 \%)$ and those with higher than secondary education $(26 \%)$. The following men subgroups had HIV screening rate higher than the national average of $6 \%$ : those aged 35-39 years $11 \%$, higher than secondary education $(18 \%)$ and richest household $(10 \%) . \mathrm{X}^{2}$ test of association showed that the following variables were significantly associated with HIV testing in both the women and men samples: age, education, marital status, religious affiliation, number of sexual partners within the last 12 months preceding the survey (wife/ husband inclusive), wealth index and the place and region of residence $(p<0.05)$. History of STI in the last 12 months was significantly associated with HIV testing in only the women sample in bivariate analysis. (table 1).

\section{Effect modification of gender on the relationship between each sociodemographic variable and HIV testing in Ghana} Gender was found to be a significant effect modifier on the relationship between HIV testing and all the sociodemographic variables under investigation : age $(p<0.001)$, marital status $(\mathrm{p}<0.001)$, household wealth index $(p<0.001)$, educational level $(\mathrm{p}<0.001)$, type of religion $(\mathrm{p}<0.001)$, total number of sexual partners in the past 12 months $(p<0.001)$, STI status in the past 12 months $(p<0.001)$, place of residence $(p<0.001)$ and region of residence $(\mathrm{p}<0.001)$ (online supplemental table 1$)$.
Gender variations in the strength of association between HIV testing and sociodemographic factors

Regressors that were important to building a statistical model to explain the variability in HIV testing were somewhat different for women and men. For the women model, seven variables were identified as the appropriate correlate of HIV testing: age, education, marital status, sexual partners in the past 12 months, diagnosed with an STI in the past 12 months, household wealth and region of residence (online supplemental table 1 and figure 1). For the men model, six regressors were identified: age, education, religion, sexual partners in the past 12 months, urban-rural residence and region of residence (online supplemental table 1 and figure 2).

These four factors are consistent correlates of HIV testing for both women and men: age, education, sexual partners in the past 12 months and region of residence (online supplemental table 1, figures 1 and 2). These consistent factors were first interpreted before the interpretation of the men or women model-specific correlates. Although the direction of the association was generally the same for most variables in both women and men, the strength of the association varied by gender in some instances.

Relative to women aged 15-19 years, the likelihood of HIV testing was about 2.1 times greater among their counterparts aged 20-24 years (APR: $2.107,95 \%$ CI: 1.513 to 2.935). A similar pattern was observed for other older age groups 25-29 years, 30-34 years, 35-39 years but not for women aged 40-44 years, and 45-49 years (online supplemental table 1 and figure 1). Unlike the women counterparts, men in the older quinary age groups had a higher likelihood of HIV testing relative to their counterparts aged 15-19 years. The magnitude of the effect was also greater in men compared with women (online supplemental table 1 and figure 2).

Compared with women with no formal education, those with higher than secondary education had a 2 times greater likelihood of undergoing HIV screening (APR: 2.026, 95\% CI: 1.510 to 2.719) (online supplemental table 1 and figure 1). A similar observation was made in the men sample but with a higher magnitude (APR: 5.735, 95\% CI: 2.464 to 13.35 ) (online supplemental table 1 and figure 2). Women who had attained secondary education were 1.3 times likely to have undergone HIV screening compared with their counterparts with no formal education (APR: 1.370, 95\% CI: 1.083 to 1.731) (online supplemental table 1 and figure 1). However, attainment of secondary school education was not a significant protective factor of HIV testing among men (online supplemental table 1 and figure 2).

Women with only one sexual partner within the last 12 months were 1.5 times likely to have undergone HIV screening compared with their counterparts who had no sexual partners (APR: $1.565,95 \%$ CI: 1.193 to 2.052) (online supplemental table 1 and figure 1). However, this segment of the men population was not significantly 


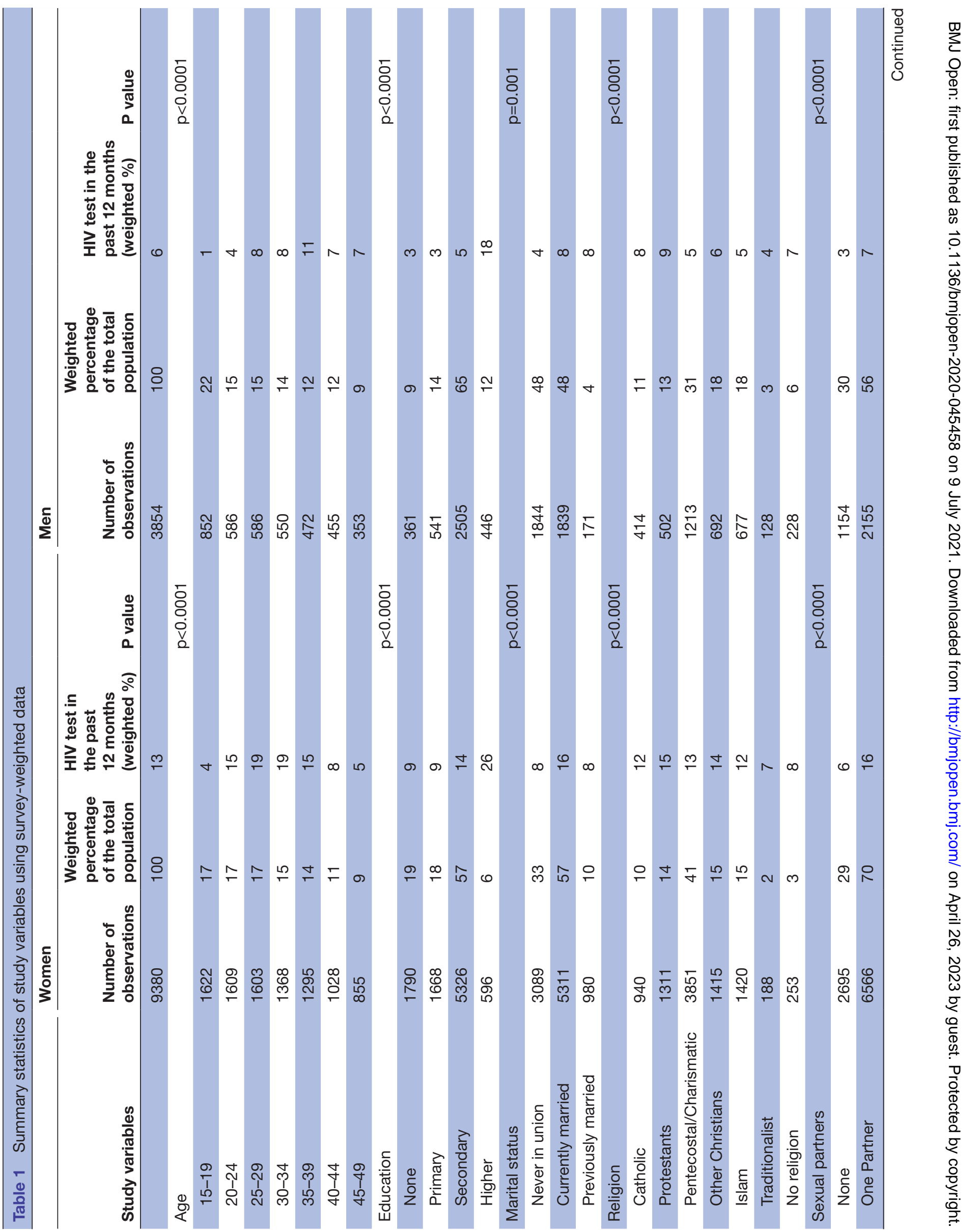




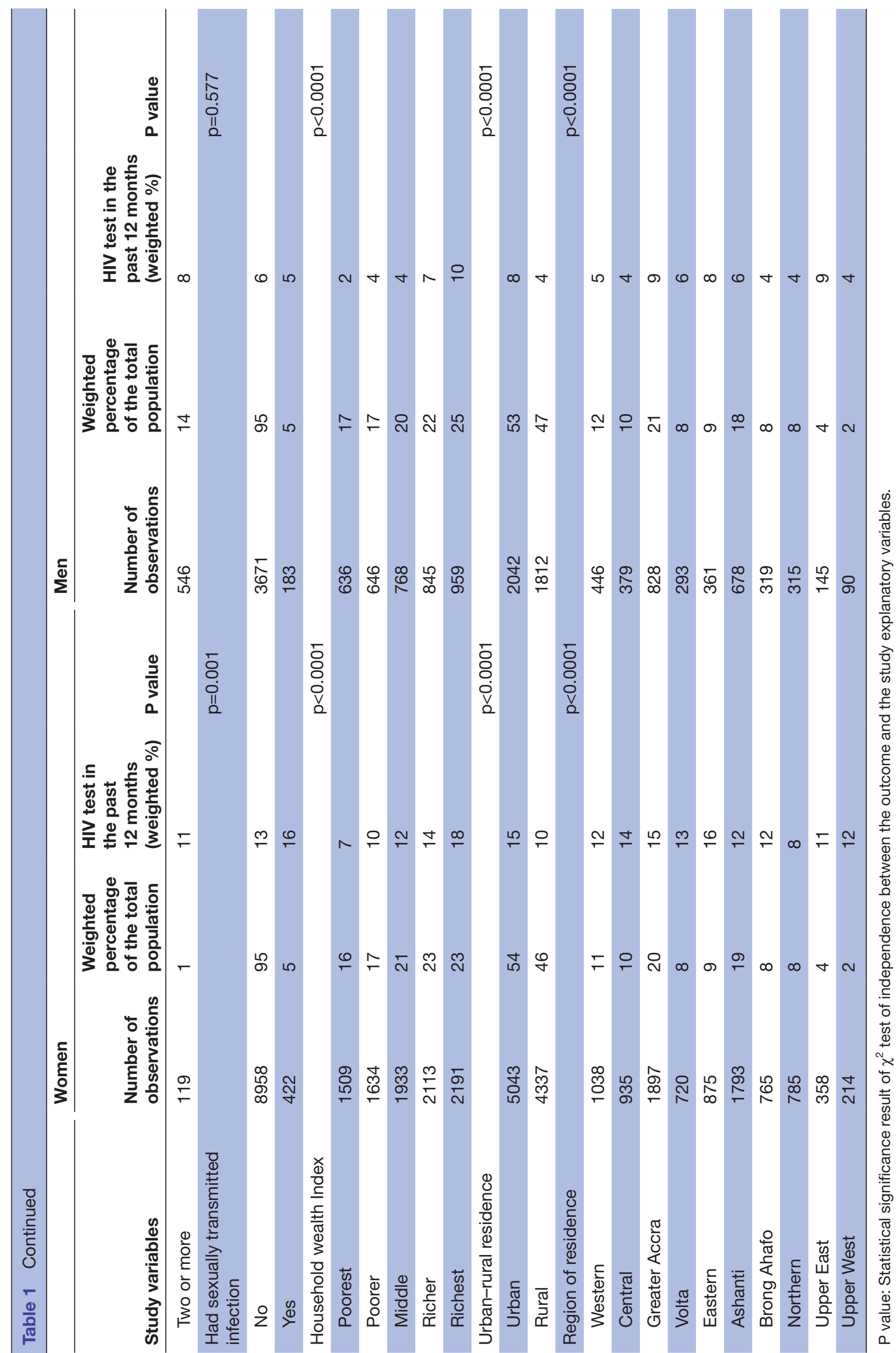

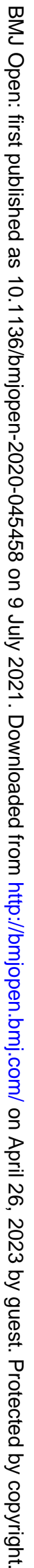




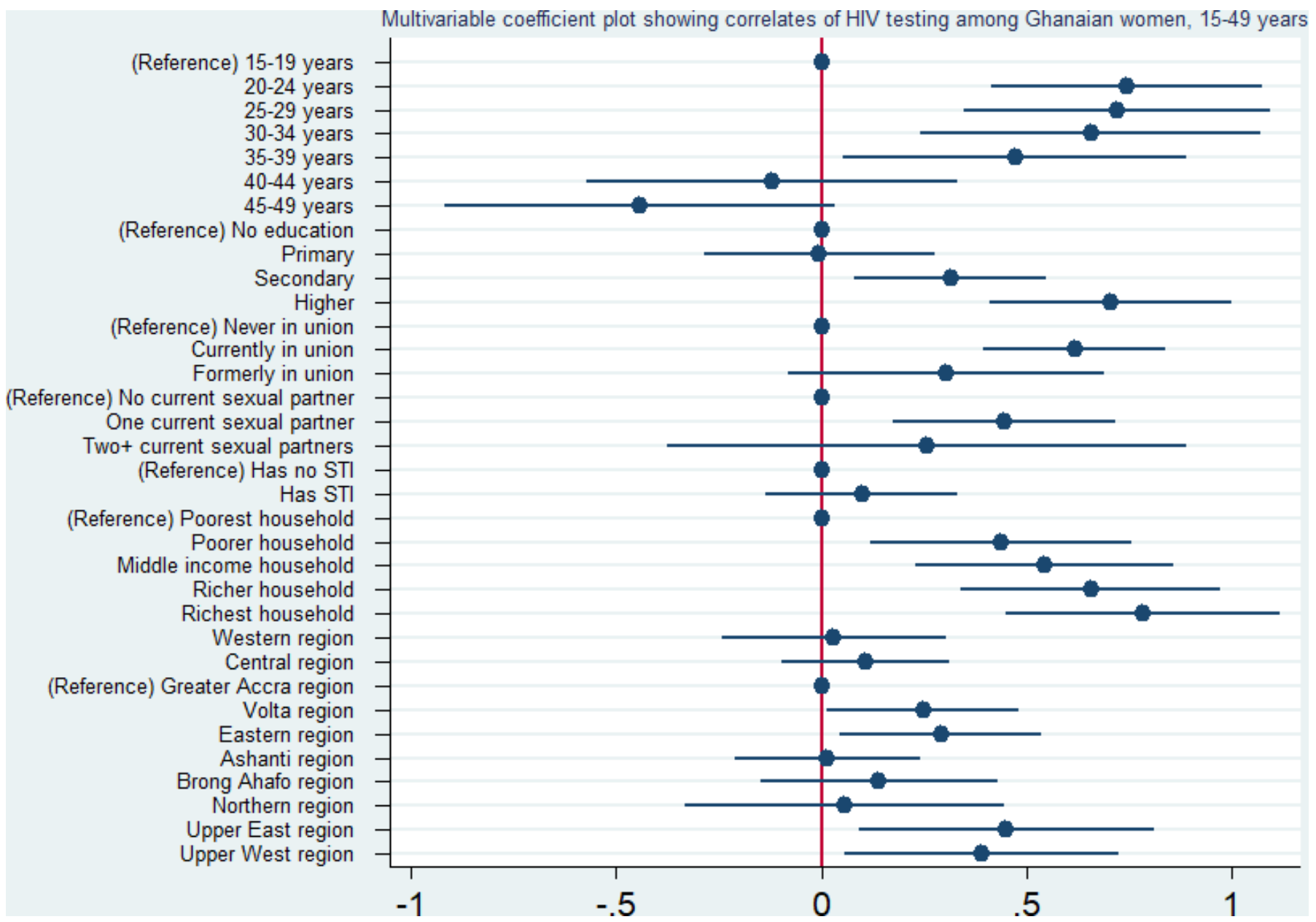

Figure 1 Coefficient plot showing the correlates of HIV testing among Ghanaian women. STI, sexually transmitted infection.

associated with HIV testing among men (online supplemental table 1 and figure 2).

Significant regional variations were observed in HIV testing among women and men. Residing in three regions was significantly associated with HIV testing compared with the greater Accra region among women, but only one region was significantly associated with the outcome among men in the same circumstances (online supplemental table 1and figures 1 and 2). For instance, compared with women living in the Greater Accra Region, those living in the Volta (APR: 1.282, 95\% CI: 1.015 to 1.619), Eastern (APR: 1.338, 95\% CI: 1.048 to 1.710 ), Upper East (APR: 1.571, 95\% CI: 1.096 to 2.253) and Upper West (APR: 1.478, 95\% CI:1.059 to 2.062) regions had a higher likelihood of testing and receiving their HIV results (online supplemental table 1 and figure 1). In the men sample, men living in the Upper East region had a 2.1 times higher likelihood of undergoing HIV screening (APR:2.188, 95\% CI: 1.187 to 4.032 ) compared with their counterparts in the Greater Accra Region (online supplemental table 1 and figure 2).

Women who were currently married were 1.8 times likely to have undergone HIV screening compared with their counterparts who have never been in union (APR: $1.855,95 \%$ CI: 1.487 to 2.313 ) (online supplemental table
1 and figure 1). The history of STI was not found to be an independent predictor of HIV screening in the women sample. Relative to the women in the poorest wealth quintiles, their counterparts who were in the poorer (APR: 1.548, $95 \%$ CI: 1.126 to 2.128 ), middle (APR: $1.724,95 \%$ CI: 1.259 to 2.360 ), richer (APR: $1.930,95 \%$ CI $: 1.406$ to 2.648) and richest (APR: $2.190,95 \%$ CI: 1.570 to 3.056 ) wealth quintiles were more likely to have undergone HIV screening in the last 12 years (online supplemental table 1 and figure 1). Religion and urban-rural residence, although were identified as important features of HIV testing among men, were not significantly associated with HIV testing in the men model (online supplemental table 1 and figure 2).

\section{HIV testing prevalence in Ghana estimated by kernel estimator approach}

Figure 3 shows the Ghana map (generated by the authors in QGIS V.3.10) with the 10 regional demarcations and labels to facilitate the interpretation of the surface maps (figure 3). Overall, the HIV testing surface maps for both women and men samples revealed that the national and regional level estimates mask subregional level variations in HIV testing (figures 4 and 5). The general observation for the surface maps of the women sample is that the 
Multivariable coefficient plot showing correlates of HIV testing among Ghanaian men, 15-49 years

(Reference) $15-19$ years
20-24 years
$25-29$ years
$30-34$ years
$35-39$ years
$40-44$ years
45-49 years

(Reference) No education

Primary

Secondary

Higher

Catholic

Protestant christians

Pentecostals/Charismatics

Other Christians

Islam

Traditionalist

(Reference) No religion/other

(Reference) No current sexual partner

One current sexual partner

Two+ current sexual partner

Urban

(Reference) Rural

Western

Central

(Reference) Greater Accra

Volta

Eastern

Ashanti

Brong Ahafo

Northern

Upper East

Upper West

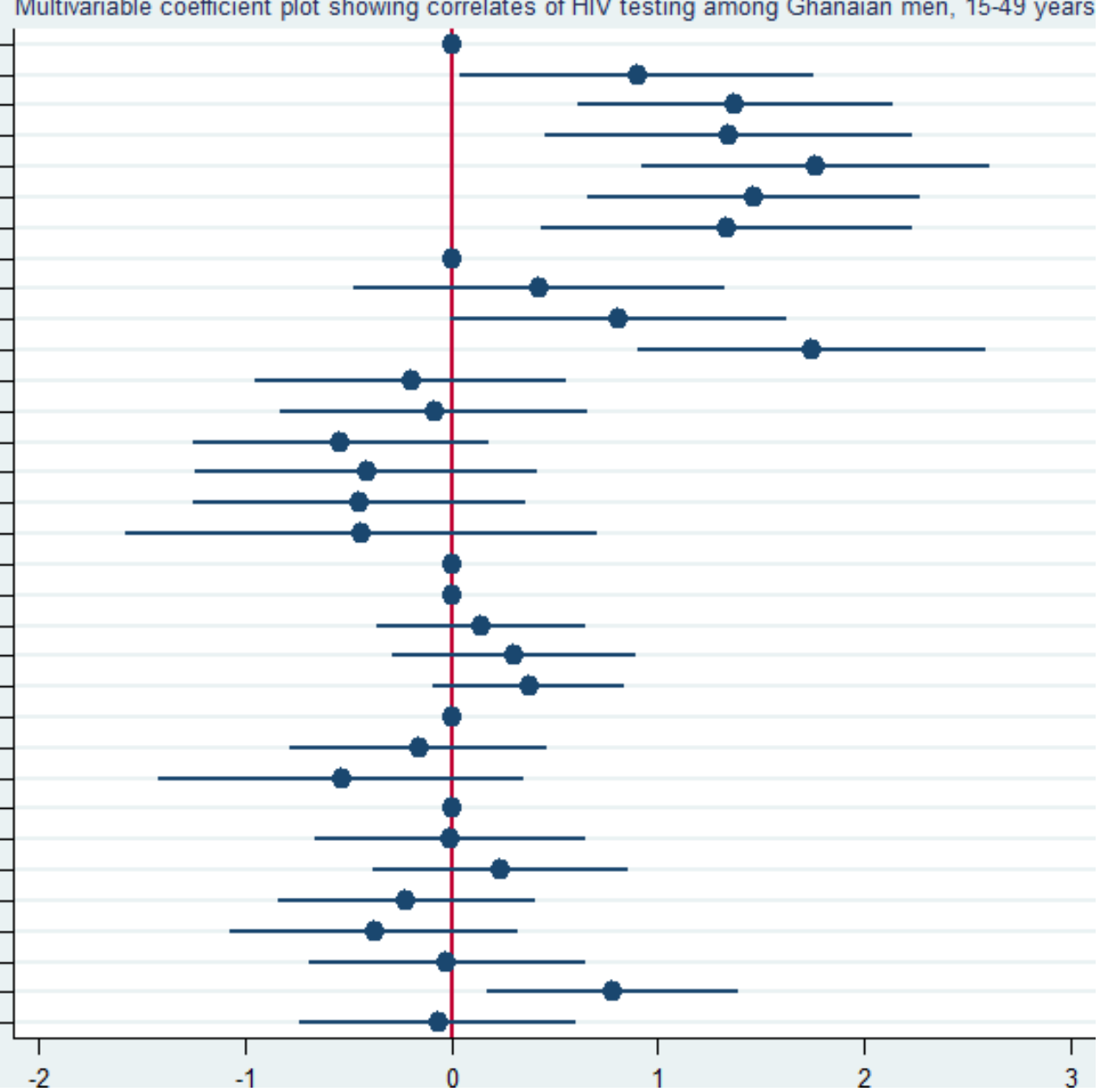

Figure 2 Coefficient plot showing the correlates of HIV testing among Ghanaian men.

areas with the highest HIV testing prevalence are in the southern regions while the lowest prevalence is found in the northern part of the country (figure 4). These regions in southern Ghana include the Eastern, Greater Accra, Western, Eastern and Volta. There are, however, areas in Upper East and West regions with relatively high HIV prevalence although the adjoining areas had low screening rate (the Northern region). For the men surface map, there were areas of high testing prevalence in southern Ghana including the adjoining areas of Eastern, Greater Accra and Volta region in the surface maps of the men sample. It also showed a high screening rate among men in some clusters in the Ashanti and Upper East Regions (figure 5). In summary, the surface maps showed interregional and intra-regional disparities in HIV screening rate in both the men and women sample. For instance, there are areas within each region with higher testing prevalence than others.

\section{DISCUSSION}

This study conducted a multivariate analysis and spatial interpolation of the predictors of HIV testing in Ghana using nationally representative data, GDHS. To the authors' knowledge, this is the first study to analyse factors that influence recent HIV test prevalence in Ghana together with spatial interpolation using the GDHS, stratified by gender. We found that the prevalence of HIV testing was higher among women (13\%) than men (6\%). Our results have shown that sex, age, education level, marital status, total sexual partners in the past 12 months, household wealth index and region of residence were significantly associated with HIV testing among women and/or men. For women, being within the age groups of 15-39 years, being currently married, attainment of postsecondary education, having only one sexual partner and dwelling in certain regions with reference to greater Accra (Volta, Eastern, Upper West and Upper East) were associated with a higher likelihood of HIV testing. For men, being older than 19 years, attainment of post-secondary education and dwelling in the Upper East region with reference to the greater Accra region were significantly associated with a higher likelihood of HIV testing.

The recent HIV testing prevalence found in our study was generally lower compared with estimates reported in Sierra Leone, Zambia, Lesotho and Zimbabwe. ${ }^{22}$ All the countries with high rates of HIV testing are located in southern Africa and the high rates of testing in these countries were attributed to high rates of HIV prevalence, 


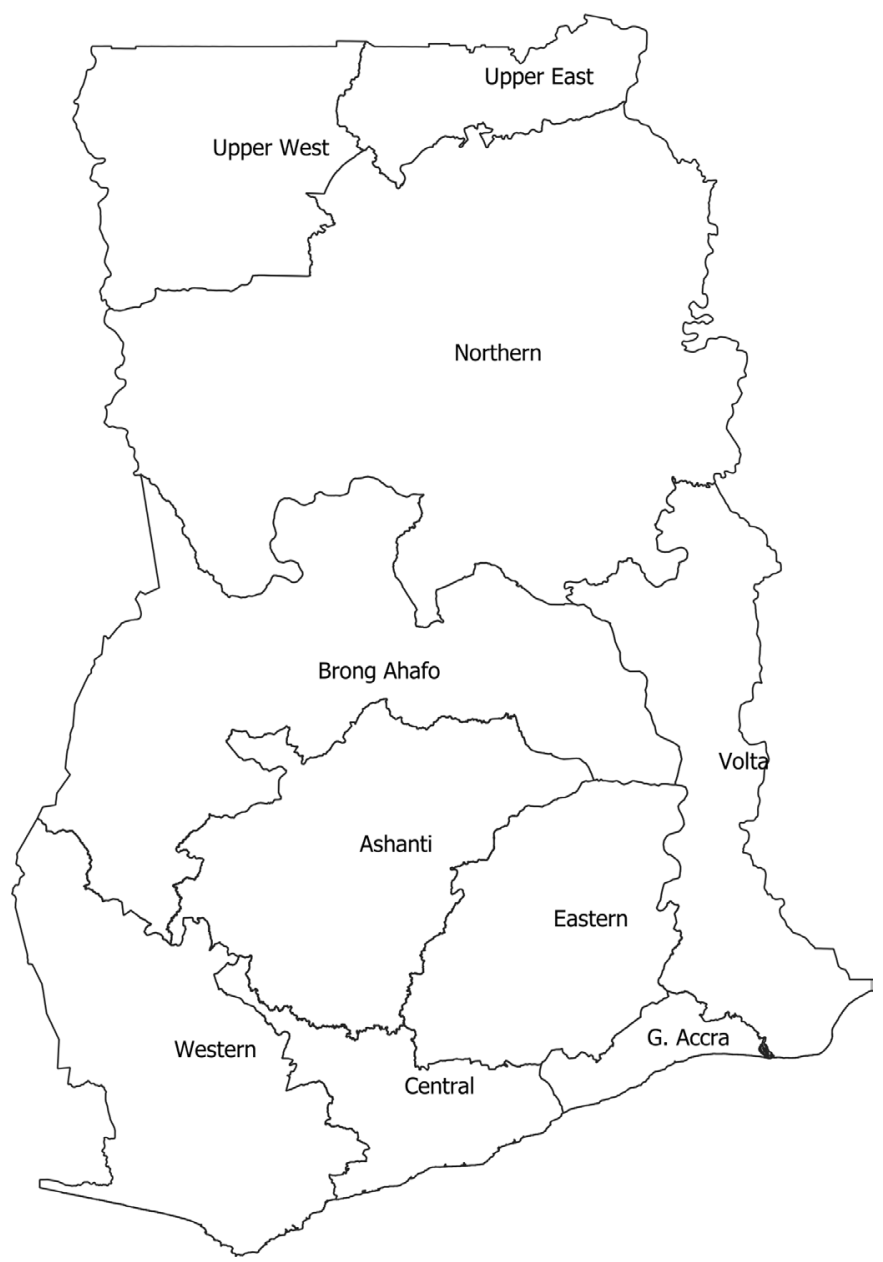

Figure 3 Ghana map showing the regional demarcation and label. G., Greater.

mandatory HIV testing at prenatal clinics and mobile clinics for HIV testing to mitigate the high prevalence of the virus. ${ }^{22}$

There are some suggestive reasons for the relatively high HIV testing in Eastern, Greater Accra, and Central among both women and men in Ghana. The districtLower Manya Krobo-that continually records the highest HIV prevalence in Ghana is in the Eastern region with a current estimate of $5.56 \% \mathrm{HIV}$ prevalence. ${ }^{23}$ Therefore, HIV reduction programmes by government and non-governmental organisations are concentrated in the Eastern region. ${ }^{24}$ HIV testing could be a proxy for access to healthcare facilities. Since the Greater Accra is the region with the highest number of healthcare facilities in Ghana, more people in the region may find it easy to screen for HIV. ${ }^{24}{ }^{25}$ Our spatial interpolation revealed that there are areas in Ghana with higher HIV testing prevalence compared with the national and regional estimates. For example, in the Western Region, regional HIV testing prevalence was $12 \%$, however, the spatial interpolation identified some clusters to be as low as $5 \%$ and some as high as $30 \%$. These within region variations underscore the importance of applying spatial interpolation in

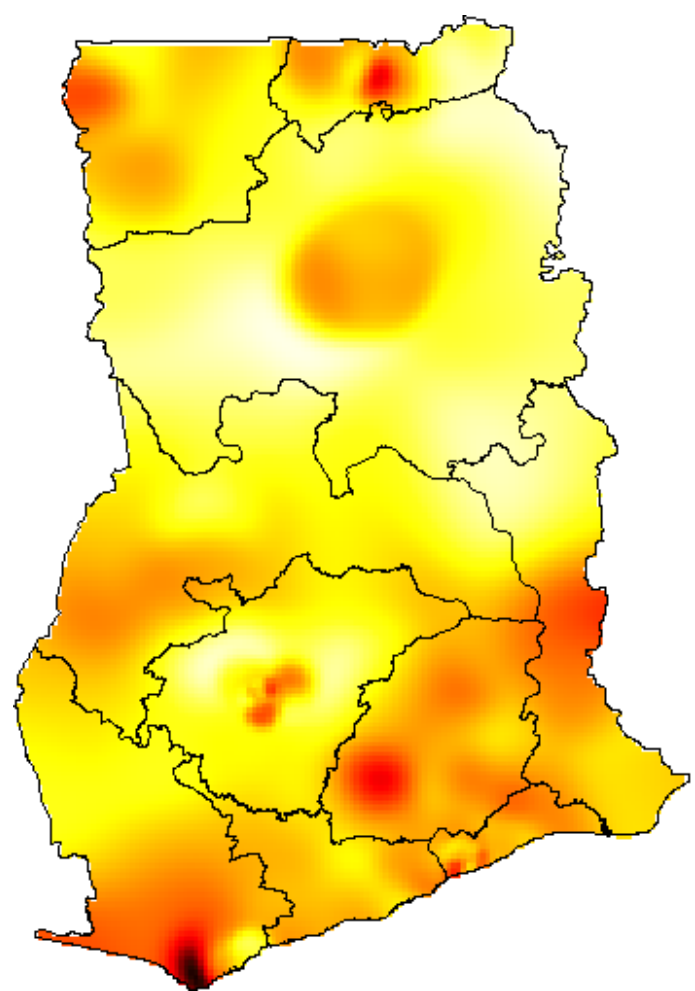

Figure 4 HIV testing prevalence among women in Ghana estimated by kernel estimator approach.

population-based studies to unmask the hidden details which can help design targeted interventions.

Women, in general, had a greater likelihood of HIV testing compared with their men counterparts, although
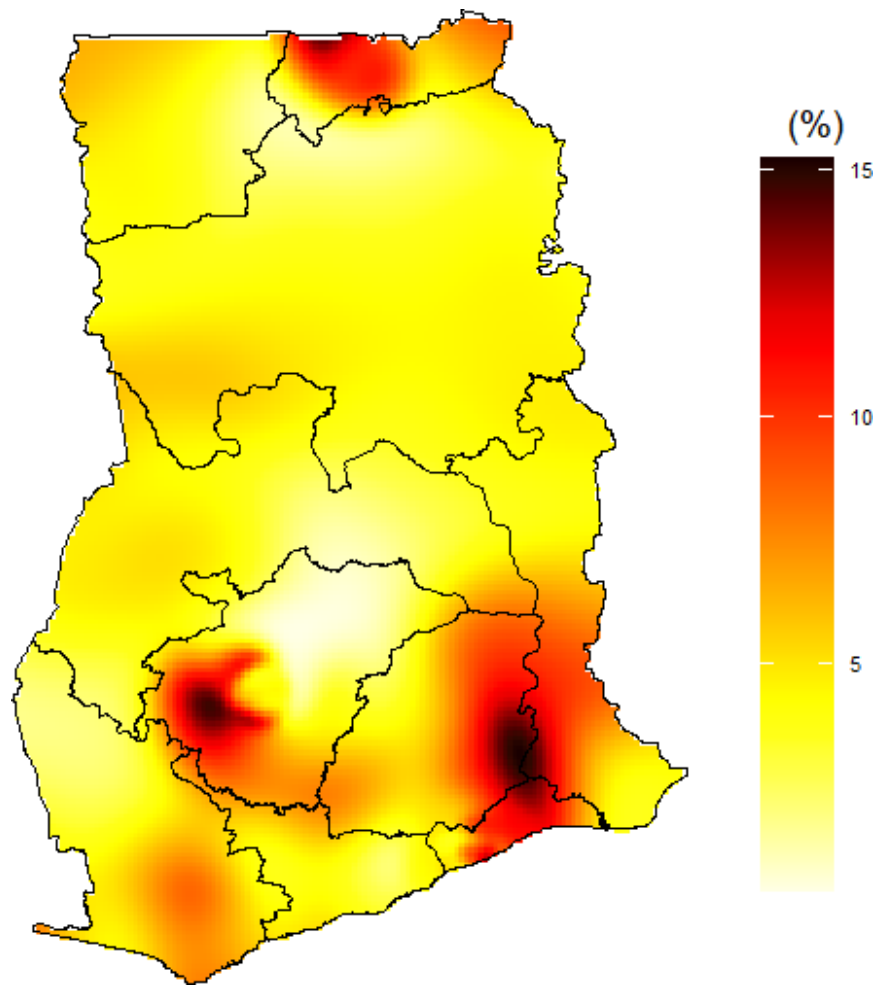

Figure 5 HIV testing prevalence among men in Ghana estimated by kernel estimator approach. 
below the national and global 90-90-90 targets, this is in agreement with previous studies. ${ }^{1126}$ This can partly be explained by the fact that HIV testing for women during prenatal care which aims at reducing mother-to-child transmission of HIV. ${ }^{27}{ }^{28}$ We found that compared with all age groups, adolescents between ages 15 and 19 years tend to have the lowest likelihood of getting tested for HIV. This finding is critical to public health because earlier studies have reported that higher rates of HIV infection among adolescents are more likely due to being engaged in risky behaviour and unsafe sex practices which put them at risk of contracting HIV. ${ }^{29}{ }^{30}$ Therefore, health policymakers should target adolescents with health education and mass HIV testing programmes and interventions. In Ghana, health testing such as breast cancer testing among older adult women has been reported to be associated with participation in club meetings. ${ }^{31}$ Given that many youth clubs exist in Ghana, they could be encouraged and incentivised to add HIV testing to their club activities.

Our results showed that educational status was a significant predictor of HIV testing among both women and men. People with higher education may have adequate knowledge and understanding of the implications of testing for HIV, thereby resulting in their likelihood to patronise testing services. Our finding concurs with previous studies, ${ }^{32} 33$ that reported a positive relationship between education and HIV testing.

We found that women who were currently married were more likely to be tested than those who have never married. For men, marital status was not significantly associated with HIV testing. Generally, in most African countries including Ghana, religious institutions and local governments require mandatory pre-marital HIV testing as a means to combat the spread of HIV among newly married couples. ${ }^{34}{ }^{35}$ Therefore, married people have the possibility of being tested for HIV compared with their unmarried counterparts. Although contentious and said to be a source of stigmatisation and human right violation to those who test HIV positive, ${ }^{34} 3637$ mandatory pre-marital HIV testing is said to be an effective way of people knowing their status. Therefore, there is a need for collaboration between public health officials and other leaders such as religious and community leaders to partner to help improve HIV testing in the communities.

Unlike men, current marriage among women was significantly associated with an increased likelihood of HIV testing. This observation can be explained by the fact that sexual activities and childbirth are socially acceptable among married couples in Ghana. ${ }^{38}$ For most women, marriage sometimes results in childbirth and HIV testing is done for all women who visit antenatal as part of the prevention of mother-to-child transmission policy in Ghana. ${ }^{39}$ This service is generally exclusive for pregnant women and does not apply to men. For currently married men, an HIV test is not required even if their wives attend antenatal care services. To encourage men to test for HIV, it is important that HIV testing is made compulsory for husbands or partners of women attending antenatal care.

Our findings also highlighted some noteworthy variations in the factors associated with HIV testing between men and women. Primary education was significantly and independently associated with an increased likelihood of HIV testing in women and men, but the effect was greater for men than women. This finding is important because it reinforces the health value of universal basic education in Ghana and the need to encourage women to attain universal basic education for improved health outcomes. Also, the magnitude of the effect of increasing age on HIV testing was generally larger for men than women. Moreover, men in age groups 40-49 years had a significantly higher likelihood of having undergone HIV testing compared with men aged 15-19 years but women in these age brackets were not significantly associated with HIV testing. This can be partly explained by the fact that most women aged 45-49 might have reached menopause and their engagement in active sexual activities might have reduced $^{40}$ and hence were probably not targeted for HIV testing by a public health professional. On the contrary, their men counterparts are generally sexually active and were more likely to have been targeted for HIV testing by public health professionals. ${ }^{41}$ We also found that women who had one sexual partner in the past 12 months were significantly more likely to undergo HIV testing, but this association was absent among men.

Another noteworthy gender variation was observed for the effect of household wealth on HIV testing. We found that wealth status predicts the likelihood of HIV testing among Ghanaian women only. Compared with the women from the poorest household, we found a higher likelihood for HIV testing among women in the poorer, middle, richer and richest households. This finding is consistent with previous studies. ${ }^{32} 3742$ The cost associated with the testing could be a barrier for people with low socioeconomic status to get tested. These findings suggest the need for sensitisation campaigns targeting communities and people with a low level of education and socioeconomic status to increase their awareness of the importance of HIV testing. This could be done through targeted outreach such as mobile clinics and integrating HIV testing into routine healthcare services, improving home-based and self-testing and subsidising the cost of the testing. Community leaders such as chiefs and religious leaders should also be involved in promoting HIV testing. This finding implies that any policy which can increase the economic standings of households, no matter how small the change may be, has a benefit on healthcare utilisation as far as HIV testing, especially for women. This finding is similar to a study conducted among the same population in Mozambique using the DHS data set. ${ }^{37}$

\section{Strength and limitation}

The current study used a large, nationally representative survey data set and employed a robust methodology for analyses. We stratified our analysis by gender which 
revealed interesting findings which can guide policy to target each gender to ensure efficiency and effectiveness of policy interventions. We employed spatial interpolation techniques that have advantages over standard statistical techniques to identify geographical variations of HIV testing prevalence in Ghana. This may be of population health significance in the effort to meet the UNAIDS 90-90-90 target not only in Ghana but in other SSA countries. Cluster analysis with the Scan Statistics method adjusts for population density and decreases selection bias as the clusters are explored without previous knowledge of their size, location or period. ${ }^{43}$ Furthermore, our study uncovered the population that is most at risk of not being tested for HIV and geographical locations with low HIV testing in Ghana. These findings could serve as a framework for public health officials to design targeted intervention to increase HIV testing.

Our findings, however, are subject to limitations that must be taken into consideration. As characteristics of all cross-sectional studies, our study could neither establish temporality nor causality of the observed association. It is important to note that all the variables in this study were self-reported and this could have introduced recall or social desirability bias. Also, we were not able to ascertain the actual reasons for participant's prior HIV testing and therefore this was not accounted for in our analysis. Moreover, a limitation with the visualisation of HIV testing using spatial maps is that the testing rate is dispersed across all pixels regardless of the presence or absence of population settlements. Despite these limitations, this study has provided profound insights from a population-level survey analysis as well as a spatial analysis of HIV testing prevalence in Ghana for informed public health action.

\section{CONCLUSION}

In conclusion, the study findings could help public health officials to better understand factors associated with HIV testing among the Ghanaian general population by showing the areas and at risk-population for targeted HIV intervention programmes. We found that $13 \%$ of women and $6 \%$ of men in the population in Ghana have ever been screened for HIV. The highest regional HIV testing prevalence was recorded in the Eastern region $(16 \%)$ and that of men was recorded in the Greater Accra region (9\%). The spatial interpolated prevalence map further revealed intra-regional level differences in HIV testing estimates. We also found gender variations in the factors associated with HIV testing which could guide policy interventions. Expansion of HIV testing, outreach through mobile clinics, home-based and self-testing, wide-ranging coverage through outreach programmes, community-based approaches and integration of opportunities or HIV testing during regular medical care is critical. These myriad approaches will be integral in reaching all persons with subclinical HIV infections in SSA for lifesaving treatment.
Author affiliations

${ }^{1}$ Family Health Care Nursing, School of Nursing, University of California San Francisco, San Francisco, California, USA

${ }^{2}$ Research Department, FOCOS Orthopaedic Hospital, Accra, Ghana

${ }^{3}$ Department of Nursing and Midwifery, University of Huddersfield School of Human and Health Sciences, Huddersfield, UK

${ }^{4}$ School of Nursing, Kwame Nkrumah University of Science and Technology, Kumasi, Ghana

${ }^{5}$ Centre for Health Policy and Implementation Research, University of Health and Allied Sciences, Ho, Ghana

${ }^{6}$ Department of Psychology, University of Ghana, Legon, Ghana

Twitter Jerry John Nutor @jjnutor, Precious Adade Duodu @Precious Adade Duodu (@PreciousDuodu) and Pascal Agbadi @PascalAgbadi

Acknowledgements We extend our gratitude to the DHS programme for granting permission to use their data for this paper.

Contributors All authors contributed to the design of the study. JJN obtained permission and downloaded data sets from the DHS programme website, and contributed to the drafting and review of the manuscript. HOD and PA contributed to the analysis of data and drafting of the manuscript. PAD, RKA and ED participated in the drafting of the manuscript. All authors critically reviewed the manuscript and approved the final version.

Funding This work was supported by the University of California, San Francisco Population Health and Health Equity fellowship programme under grant number 7504575 .

Competing interests None declared.

Patient consent for publication Not required.

Ethics approval Ethical approval for the 2014 Ghana DHS data collection was obtained from the Ethical Review Committee of the Ghana Health Service and the Institutional Review Board of ICF International.

Provenance and peer review Not commissioned; externally peer reviewed.

Data availability statement Data are available in a public, open access repository. The 2014 GDHS data are publicly available upon a simple registration-access request so data used for this study can be obtained from the DHS website at https://dhsprogram.com/data/dataset_admin/index.cfm.

Supplemental material This content has been supplied by the author(s). It has not been vetted by BMJ Publishing Group Limited (BMJ) and may not have been peer-reviewed. Any opinions or recommendations discussed are solely those of the author(s) and are not endorsed by BMJ. BMJ disclaims all liability and responsibility arising from any reliance placed on the content. Where the content includes any translated material, BMJ does not warrant the accuracy and reliability of the translations (including but not limited to local regulations, clinical guidelines, terminology, drug names and drug dosages), and is not responsible for any error and/or omissions arising from translation and adaptation or otherwise.

Open access This is an open access article distributed in accordance with the Creative Commons Attribution Non Commercial (CC BY-NC 4.0) license, which permits others to distribute, remix, adapt, build upon this work non-commercially, and license their derivative works on different terms, provided the original work is properly cited, appropriate credit is given, any changes made indicated, and the use is non-commercial. See: http://creativecommons.org/licenses/by-nc/4.0/.

\section{ORCID iDs}

Jerry John Nutor http://orcid.org/0000-0002-7562-6281

Henry Ofori Duah http://orcid.org/0000-0002-4842-6006

Pascal Agbadi http://orcid.org/0000-0001-5297-2512

\section{REFERENCES}

1 World Health Organization. HIV/AIDS: data and statistics, 2018. Available: https://www.who.int/hiv/data/en/

2 UNAIDS. Global HIV \& AIDS statistics - 2019 fact sheet, 2019. Available: https://www.unaids.org/en/resources/fact-sheet

3 World Health Organization. HIV/AIDS Fact Sheet Geneva: World Health Organization 2019 [updated November 15, 20192019]. Available from. Available: https://www.who.int/en/news-room/factsheets/detail/hiv-aids [Accessed November 25 2019]. 
4 Kharsany ABM, Karim QA. Hiv infection and AIDS in sub-Saharan Africa: current status, challenges and opportunities. Open AIDS J 2016;10:34-48

5 Narin P, Yamamoto E, Saw YM, et al. Factors associated with HIV testing among the general male population in Cambodia: a secondary data analysis of the demographic health survey in 2005 , 2010, and 2014. PLoS One 2019;14:e0219820.

6 UNAIDS. Aids JUNPo: 90-90-90: an ambitious treatment target to help end the AIDS epidemic. Geneva: UNAIDS, 2014.

7 Asamoah CK, Asamoah BO, Agardh A. A generation at risk: a crosssectional study on HIV/AIDS knowledge, exposure to mass media, and stigmatizing behaviors among young women aged 15-24 years in Ghana. Glob Health Action 2017;10:1331538.

8 Gómez-Ayerbe C, Martínez-Sanz J, Muriel A, et al. Impact of a structured HIV testing program in a hospital emergency department and a primary care center. PLoS One 2019;14:e0220375.

9 Koku EF, Stigma KEF. Stigma, sexual risk and desire for HIV tests in Ghana. Sex Health 2011;8:110-9.

10 Laar A, DeBruin D. Key populations and human rights in the context of HIV services rendition in Ghana. BMC Int Health Hum Rights 2017;17:1-10.

11 Ali H, Amoyaw F, Baden D, et al. Ghana's HIV epidemic and PEPFAR's contribution towards epidemic control. Ghana Med J 2019;53:59-62.

12 Ayisi Addo S, Abdulai M, Yawson A, et al. Availability of HIV services along the continuum of HIV testing, care and treatment in Ghana. BMC Health Serv Res 2018;18:739.

13 van Hulst M, Sagoe KWC, Vermande JE, et al. Cost-Effectiveness of HIV screening of blood donations in Accra (Ghana). Value Health 2008;11:809-19.

14 Nutor JJ, Duodu PA, Agbadi P, et al. Predictors of high HIV+ prevalence in Mozambique: a complex samples logistic regression modeling and spatial mapping approaches. PLoS One 2020;15:e0234034.

15 Osei-Yeboah J, Lokpo SY, Ussher FA, et al. The epidemiology of human immunodeficiency virus (HIV) and syphilis in Ghana: a fiveyear single urban site parallel population-based analysis vis-à-vis the sentinel survey. J Trop Med 2018;2018:1-7.

16 GSS G. Ghana statistical service (GSS), Ghana health service (GHS), and ICF macro. Accra: Ghana Demogr Health Surv 2009;2008:79-96.

17 Lindsey C, Sheather S. Best subsets variable selection in nonnormal regression models. Stata J 2015;15:1046-59.

18 Furnival GM, Wilson RW. Regressions by leaps and bounds. Technometrics 2000;42:69-79.

19 Lawless JF, Singhal K. Efficient screening of Nonnormal regression models. Biometrics 1978;34:318-27.

20 Larmarange J, Vallo R, Yaro S, et al. Methods for mapping regional trends of HIV prevalence from demographic and health surveys (DHS. Cybergeo: European Journal of Geography, 2011.

21 R Core Team. R: a language and environment for statistical computing 2015 https://www.R-project.org

22 Staveteig S, Croft TN, Kampa KT, et al. Reaching the 'first 90': Gaps in coverage of HIV testing among people living with HIV in 16 African countries. PLoS One 2017;12:e0186316.

23 Ghana AIDS Commission. National HIV and AIDS policy: universal access to HIV prevention, treatment and care services towards ending AIDS as a public health threat. Accra, Ghana, 2019. Available: https://www.ghanaids.gov.gh/mcadmin/Uploads/nationalHIVandAI DSPolicy(2).pdf

24 Hushie M. Public-non-governmental organisation partnerships for health: an exploratory study with case studies from recent Ghanaian experience. BMC Public Health 2016;16:963.
25 Tun W, Okal J, Schenk K, et al. Limited accessibility to HIV services for persons with disabilities living with HIV in Ghana, Uganda and Zambia. J Int AIDS Soc 2016;19:20829.

26 Sanga Z, Kapanda G, Msuya S, et al. Factors influencing the uptake of voluntary HIV counseling and testing among secondary school students in Arusha City, Tanzania: a cross sectional study. BMC Public Health 2015;15:452.

27 Nyuzaghl J, Ohene S, Odoi-Agyarko K. Acceptability of routine offer of HIV testing (opt-out approach) among pregnant women in the WA municipality. Ghana Med J 2011;45:10-5.

28 Camlin CS, Ssemmondo E, Chamie G, et al. Men "missing" from population-based HIV testing: insights from qualitative research. AIDS Care 2016;28 Suppl 3:67-73.

29 Dellar RC, Dlamini S, Karim QA. Adolescent girls and young women: key populations for HIV epidemic control. J Int AIDS Soc 2015;18:19408

30 Kim YK, Small E, Okumu M. School-Based HIV/AIDS education, risky sexual behaviors, and HIV testing among high school students in the United States. Soc Work Health Care 2019;58:258-73.

31 Agyemang AF, Tei-Muno AN, Dzomeku VM, et al. The prevalence and predictive factors of breast cancer screening among older Ghanaian women. Heliyon 2020;6:e03838.

32 Lépine A, Terris-Prestholt F, Vickerman P. Determinants of HIV testing among Nigerian couples: a multilevel modelling approach. Health Policy Plan 2015;30:579-92.

33 Mirandola M, Gios L, Joanna Davis R, et al. Socio-Demographic factors predicting HIV test seeking behaviour among MSM in 6 EU cities. Eur J Public Health 2017;27:313-8.

34 Akoku DA, Tihnje MA, Tarh EO, et al. Predictors of willingness to accept pre-marital HIV testing and intention to sero-sort marital partners; risks and consequences: findings from a population-based study in Cameroon. PLoS One 2018;13:e0208890.

35 Barmania S, Aljunid SM. Premarital HIV testing in Malaysia: a qualitative exploratory study on the views of major stakeholders involved in HIV prevention. BMC Int Health Hum Rights 2017;17:1-10.

36 Ifemeje S. Mandatory premarital HIV testing policy in Nigeria: a gross violation of the rights of people living with HIV/AIDS. The International Journal of Human Rights 2012;16:401-10.

37 Agbadi P, Nutor JJ, Darkwah E, et al. Covariates and spatial interpolation of HIV screening in Mozambique: insight from the 2015 AIDS indicator survey. Int J Environ Res Public Health 2020;17:5630.

38 Elleamoh GE, Dake FAA. "Cementing" marriages through childbearing in subsequent unions: Insights into fertility differentials among first-time married and remarried women in Ghana. PLoS One 2019:14:e0222994.

39 Ejigu Y, Tadesse B. HIV testing during pregnancy for prevention of mother-to-child transmission of HIV in Ethiopia. PLoS One 2018;13:e0201886.

40 Krasnow SS, Maglio A-S. Female sexual desire: what helps, what hinders, and what women want. Sexual and Relationship Therapy 2019;15:1-29.

41 Gewirtz-Meydan A, Ayalon L. Why do older adults have sex? Approach and avoidance sexual motives among older women and men. J Sex Res 2019;56:870-81.

42 Agha S. Factors associated with HIV testing and condom use in Mozambique: implications for programs. Reprod Health 2012;9:20.

43 Chimoyi LA, Musenge E. Spatial analysis of factors associated with HIV infection among young people in Uganda, 2011. BMC Public Health 2014;14:555. 